\title{
Design of Orchard Monitoring Node Based on ZigBee Technology
}

\author{
Na Xie \\ Xian yang Vocational and Technical College, College of Electronic Information, 712000,China
}

\begin{abstract}
Keywords: Orchard Environment, ZigBee, Environmental Monitoring.
\end{abstract}
\begin{abstract}
With the development of science and technology, in order to ensure the sustainable and healthy development of fruit industry economy, the goal is to develop agricultural informatization and deep agriculture. Analysis of the orchard environment information, analysis of the orchard environment, analysis of the growth of fruit trees. By analyzing the growth of fruit trees, reasonable irrigation and fertilization can minimize the maintenance cost of the orchard and increase the fruit harvest. In the past, orchard management can only manually collect environmental information such as temperature, humidity and light from orchard, which can not guarantee the real-time and accuracy of data. In this draft, the orchard monitoring node based on ZigBee technology is designed. In order to ensure the real-time and data reliability of data transmission, the corresponding information management system is developed. The system can display the environmental information around the orchard in time, and users can make decisions according to the environmental information.
\end{abstract}

\section{Introduction}

China has a long history in planting fruit trees and is one of the world's recognized fruit producing areas. Since the reform and opening-up in 1978, agriculture has provided important policy support for agricultural policy, and the science and technology in the field of agriculture have made progress. In this case, China's fruit varieties are very rich, and the fruit industry has developed. Relevant statistics: since 1993, there have been fruit producing areas and various types of fruit trees all over the world. In addition to the advantages of yield and planting area, the quality of fruit cultivated in China has also been greatly improved. At present, the fruit industry has become the third largest agricultural planting industry in China. Internationally, fruit planting is a major industry in China. Fruit industry has become a pillar industry of local economy in many areas[1]. At the same time, the fruit industry has brought considerable income to farmers. And it became the source of their wealth. With the improvement of people's living standards, the demand for fresh and harmless high-grade fruits is increasing, the demand for fruits is diversified, and high-quality fruits have great market potential. In order to ensure the sustainable and healthy development of fruit industry economy, the development of agricultural information, the development of precision agriculture, the timely and effective response of orchard soil information and planting fruit tree information is necessary. It is used to analyze the environment of orchard and the growth of fruit trees[2]. The growth of fruit trees, the utilization of water resources, and the rational irrigation and fertilization of fertilizers minimize the production and maintenance costs of fruit trees, increase fruit yield and reduce environmental pollution.

\section{Overall Design of Orchard Monitoring System}

\subsection{System Architecture}

The orchard environment acquisition system collects the basic information of the orchard environment in real time through the real-time sensor, and collects the temperature, humidity and light quantity around the orchard through the real-time sensor. The information is transmitted to the upper computer system in real time through the convergence node. The other is the orchard information processing system[3]. Data is collected, processed by ZigBee network coordinator, and then sent remotely in GPRS module. The remote data receiving program receives the information 
and sends it to the information processing system for processing.

\subsection{Wireless Sensor Network}

Multiple sensor nodes reported to the user transmit detection data to the host node and transmit it to the user through other networks. In this definition, wireless sensor network is usually composed of three main parts: sensor node, sink node and server management center. After the nodes expand, the surrounding data is collected by the sensor nodes and self-organized through the network. Parameter setting can be used as network path to complete data transmission task. The data collected through the multi node self-organized network is mainly sent to the host node in a multi hop way. The host node can send data to the server management center through an effective longdistance link for further processing and analysis[4]. The system usually consists of four parts. Data acquisition department is responsible for collecting and sending data through communication serial port protocol. The data processing unit is a terminal node core mainly composed of embedded chips. This is mainly due to the specific operation of the terminal node and the processing of the data transceiver. The vehicle antenna or transmission module is mainly used for long-distance and shortdistance data transmission. The power supply part is the reason for the operation of the terminal node of the stable power supply. Public power sources are solar and lithium batteries. At the same time, sensor networks have large-scale, strong self-assembly ability, high dynamic, security and reliability. Therefore, the application of wireless sensor network in the fields of industrial agriculture, military security, environmental protection and medical treatment has greatly improved human life.

\subsection{Remote Data Interaction}

General packet broadcast service. GPRS has continuous on-line function and charges based on the amount of data sent. When the terminal is connected with GPRS network, the terminal continues to connect with the network. Without data transmission, there is no charge for transportation cost[5]. GPRS network uses packet switching technology which can support multiple bandwidth, has high data transmission rate, and can effectively use limited bandwidth resources. In real-time transmission, network service ensures the real-time nature of data exchange and is occupied only during transmission. Therefore, GPRS network can reduce the cost of network maintenance.

\subsection{Zigbee Network Equipment Type}

In ZigBee network, according to the function of network equipment, it can be divided into full function equipment and reduced function equipment. Pure functional component: in the network, the network acts as the coordinator, which is the function of the controller. Any ZigBee device and communication can have any network topology, and support all functions and functions specified in the IEEE 802.15.4 standard. A fully functional device can act as a router on the network when it is idle. Reduced functional devices: responsible for regular use of data collection terminal devices in the network, which is not available for network coordinator or router, and then interstellar topology can only be used. The cost of RFD node is relatively low, the function is less, the implementation is relatively simple and requires relatively small protocol space. In addition, RFID nodes can not directly communicate with each other through FFD nodes. According to the types of network nodes, there are three types of logic: coordinator, router and terminal device. Coordinator: a coordinator is an FFD device. Select the channel and network identifier[6]. It is the central node of the network, responsible for the start-up and configuration of the network. This is to send the network beacon and start the whole network. Only coordinators are allowed on the network. After the whole network is started and constructed, it manages information equivalent to other nodes of the router. It continuously receives information from other nodes and communicates with other modules by connecting serial ports. Therefore, the coordinator node must be in the active state of continuous power supply. 


\section{Communication Distance Test}

Through ti's packet filtering software, we can test the packet transmission between nodes. Through the test, the maximum transmission distance of $2530 \mathrm{CC}$ and $2530 \mathrm{CC}$, the full power transmission reaches the setting of 4.5 DBM. In addition, 50 nodes were used in the experiment[7]. 25 experiments in different places, two wireless transmission nodes in each group. The details of data packets are recorded by continuously increasing the transmission distance. Analyze the data analysis of the software and calculate the average value in order to prepare the data analysis chart.

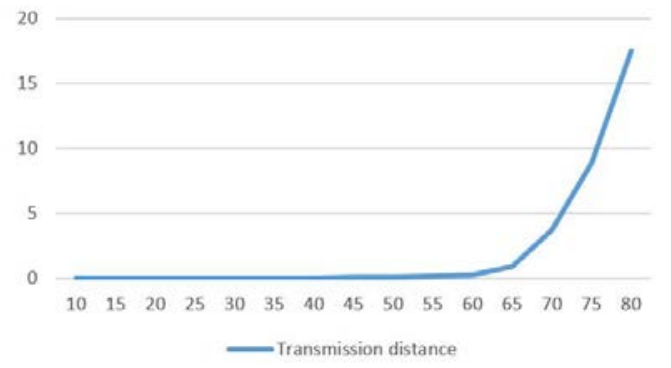

Figure 1 Test results of communication distance

\section{System Networking Test}

The whole orchard is about $225 \mu \mathrm{m}$ in area, $4500 \mathrm{~m}$ in length and $350 \mathrm{~m}$ in width, which is rectangular. There are 5 peach varieties in different ripening stages. In order to expand the monitoring nodes of the whole orchard, the field survey was carried out with the orchard as the object, and the corresponding network deployment plan was formulated.

In the above structure, the square side is $60 \mathrm{~m}$ long, and the number of router nodes in the whole orchard will be 29 in the future. Coordinator nodes are represented by boxes, so router nodes are represented by circles in the diagram[8]. The remaining end nodes are assigned to the entire orchard. Through the actual orchard, the deployment is generally based on the orchard monitoring deployment structure. According to the actual deviation of fruit tree distance, the corresponding adjustment was made. Perform power and network tests on the device, analyze whether the coordinator is connected to the serial port and send data through the software. Due to the relatively large number of network nodes, the data transmission interval of the nodes is set to 0 to 5 minutes through the configuration parameters. By designing 8-byte transmission information, the data transmission status of the monitored nodes in the whole system is analyzed. After 120 hours of continuous system operation, the transmission hop and packet loss rate of nodes are analyzed.

In the whole orchard expansion, the node can reach up to 6 coordinator nodes. In the continuous operation of the system, the average maximum packet loss rate is $1.9 \%$, which can meet the general application range[9]. According to the above figure, in order to improve the transmission success rate, we must reduce the transmission distance, increase the number of nodes, and increase the cost of node expansion. Therefore, by slightly reducing transmission reliability, the cost of system implementation can be reduced.

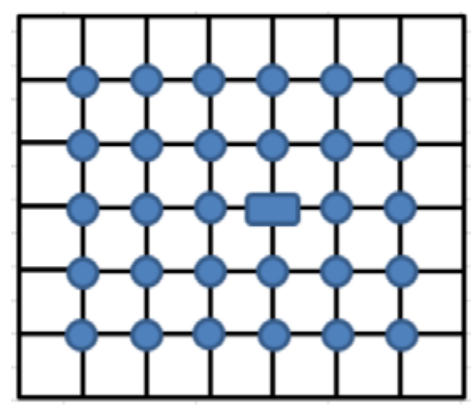

Figure 2 Orchard monitoring node deployment 


\section{Database Design}

A database designed to store collected information about related information. The terminal sending information of storage coordinator is mainly composed of terminal node information table, table, abnormal data, real-time monitoring data abnormal alarm, table, resume data, abnormal data resume, etc. through lever. Table. The real-time monitoring data table is mainly used to store the data sent by the coordinator and stored in the monitoring data table according to the receiving time. Due to the large amount of data stored in the real-time monitoring data table, each month is divided into 12 tables, which are stored in different tables and registered in the data table regularly. When the amount of data is huge, you can back up the data and clear the historical data table.

\section{Conclusion}

In the traditional orchard environment monitoring system based on ZigBee technology, most of them rely on manual collection in order to obtain the temperature, humidity, light and other environmental information of the orchard timely and effectively. This is a comparison of technology and precision. The purpose of this study is to understand the development of environmental monitoring at home and abroad, the corresponding technology applications and the corresponding hardware node design. Determine the collection conditions of the whole orchard, and formulate the overall planning of the orchard monitoring system. Understand the characteristics of wireless sensor network, analyze the advantages and disadvantages of different wireless network technologies. In particular, please analyze ZigBee protocol stack structure, ZigBee network device type and ZigBee network topology. By determining the overall request, the main control chip, sensor and GPRS module are selected. By understanding the working principle of the sensor and the main control chip, the related peripheral drive circuit is designed. In order to collect the temperature, humidity and light information around the orchard, the corresponding software design was carried out. Completed the corresponding orchard information processing system, completed the node transmission distance and node network deployment test.

\section{Acknowledgements}

Project No.: 2019k02-08

Project Name: Development and application of dynamic monitoring and imaging system for fruit tree growth cycle based on Data Mining

Project category: special research project of science and Technology Research Bureau

\section{References}

[1] Wu, Fengbo., Lv, Xitong., Zhang, Huike. Design and Development of Forest Fire Monitoring Terminal // 2018 International Conference on Sensor Networks and Signal Processing (SNSP), 2018.

[2] Hang, Ji., Liechang, Miao., Jianfang, Dong. Indoor Environment Remote Monitoring System Based on NB-IOT. IOP Conference Series Materials Science and Engineering, vol. 452, no. 2, pp. 022162, 2018.

[3] WANG, Hao., ZHANG, Xuefei., SUN, Xiang,. Research and Experiment on Performance Stability of Facility Greenhouse Environmental Monitoring System. Asian Agricultural Research, 10, 2018.

[4] Hong, H., Hou, Y., Zhang, Z. Research on indoor environment monitoring system based on ZigBee, 2017.

[5] Mu, J., Liang, H. Performance analysis of the ZigBee networks in 5G environment and the nearest access routing for improvement, 2017. 
[6] Xingxing, Liu., Tong, Zhang., Baosheng, Li,. Wireless Measurement and Control System of Environmental Parameters in Greenhouse Based on ZigBee Technology, 2018.

[7] Zhang, Cong-guang., Han, Jian-cong., Qiu, Ling., et al. Energy method-based evaluation of the sustainability of the "Five-in-One" ecological orchard system, 2018.

[8] Zhang, Cong-guang., Han, Jian-cong., QIU, Ling., et al. Energy method-based evaluation of the sustainability of the "Five-in-One" ecological orchard system, 2018.

[9] Simon, M.Bryant., Cherrie, H.T.Kong., Mark, B. Cannell,. Loss of caveolin-3-dependent regulation of ICa in rat ventricular myocytes in heart failure. American Journal of Physiology Heart \& Circulatory Physiology, vol. 314, no. 3, 2017. 